\title{
Comportement mécanique d'un acier au carbone sous l'effet du brunissage ou du galetage
}

\author{
Hamid Hamadache ${ }^{1, a}$, Lakhdar Laouar ${ }^{2}$ et Kamel Chaoui ${ }^{1}$ \\ 1 Laboratoire LR3MI, Département de Mécanique, Université Badji Mokhtar, BP 12, Annaba 23000, Algérie \\ 2 Département de Mécanique, Université Badji Mokhtar, BP 12, Annaba 23000, Algérie
}

Reçu le 17 janvier 2006, accepté le 19 février 2007

\begin{abstract}
Résumé - Les pièces travaillant en contact finissent par se dégrader par suite du phénomène d'usure. De ce fait, elles exigent un matériau à hautes caractéristiques des couches superficielles et une gamme de fabrication très poussée. Le galetage et le brunissage peuvent conférer au matériau un nouvel aspect de surface tant sur le plan micro-géométrique que physique. Ce travail a pour objectif de préciser par comparaison, les effets des deux procédés. Par ailleurs les influences des divers paramètres du régime de formage sur la rugosité et sur la dureté ont été étudiées. Les résultats obtenus montrent que le nouvel aspect de surface requis est d'un indice technico-économique appréciable. Les couches superficielles traitées se comportent à la fois comme des surfaces rectifiées $\left(R_{\mathrm{a}}=0,22\right.$ à $\left.0,9 \mu \mathrm{m}\right)$ et écrouies $(H R B=87$ à 90). Ces effets optimaux se déduisent d'un régime de travail spécifique à chaque caractéristique et à chaque procédé. Ceci permet une amélioration d'environ $98 \%$ de la tenue à l'usure de ces couches et de les classer comme des surfaces rodées.
\end{abstract}

Mots clés : Usinage / rugosité / dureté / usure / brunissage / galetage

\begin{abstract}
Mechanical behaviour of carbon steel under ball and roller burnishing effect. When working in contact, some machine components are subjected to damage following wear phenomenon. Therefore, high mechanical properties material is requested and developed manufacturing processes are necessary. Ball and roller burnishing, which are essential in cold-forming processes, lead to better geometrical and physical surface qualities. The optimal effect of each of these two processes is conditioned by specific regime parameters for each desired characteristic. The aim of this paper is to compare the effect of both processes. The relative influence of the different parameters on roughness and hardness is considered. Results show that the new state of the formed superficial layers presents a good technical and economic index when comparing to conventional production process. The superficial layers generated by ball and roller burnishing can be considering as a hardened $(\mathrm{HRB}=87$ to 90$)$ and grinded $\left(R_{\mathrm{a}}=0.9\right.$ to $\left.0.22 \mu \mathrm{m}\right)$ surface. Thence, the measurements revealed a wear resistance improvement of $98 \%$. Therefore, the burnished surface behavior may be classified as runned-in surfaces.
\end{abstract}

Key words: Machining / roughness / hardness / ball burnishing / roller burnishing

\section{Introduction}

Pour assurer la finition d'une pièce, on recourt très souvent à une série d'usinage de finition [1] moyennant parfois un prix de revient élevé. Par ailleurs, les couches superficielles, étant les plus sollicitées en service, sont donc les premières à se dégrader par suite des différentes actions de contact aussi bien avec les pièces

\footnotetext{
a Auteur pour correspondance : hamham36@yahoo.fr
}

conjuguées qu'avec le milieu environnant. En contact, sous l'effet du frottement celles-ci se dégradent par suite d'usure $[2,3]$. Actuellement $50 \%$ de l'énergie fournie est perdue par frottement résultant des mouvements relatifs entres les éléments de machines. De nos jours, les opérations de parachèvement et de renforcement des matériaux sévèrement sollicités font appel à des procédés modernes impliquant un traitement mécanique tels que le galetage [4-6] ou le brunissage [7-9]. Ces deux procédés mettent en ouvre une déformation plastique à froid des 


\section{Nomenclature}

\begin{tabular}{|c|c|}
\hline$A \%$ & Allongement à rupture (\%) \\
\hline$d_{\mathrm{G}}$ & Diamètre du galet (mm) \\
\hline$f$ & Vitesse d'avance de formage $\left(\mathrm{mm} \cdot \mathrm{tr}^{-1}\right)$ \\
\hline$H R B$ & Dureté Rockwel échelle B \\
\hline$i$ & Nombre de passes en formage (passes) \\
\hline$m_{0}$ & Masse initiale de la pièce avant l'essai (g) \\
\hline$m_{i-1}$ & Masse de la pièce après l'essai antérieur (g) \\
\hline$m_{i}$ & Masse de la pièce après l'essai en cours (g) \\
\hline$n$ & Nombre de tours de la broche $\left(\operatorname{tr} . \min ^{-1}\right)$ \\
\hline$P$ & Effort de formage (daN) \\
\hline$R_{\mathrm{a}}$ & Rugosité moyenne arithmétique $(\mu \mathrm{m})$ \\
\hline$R_{\mathrm{e}}$ & Limite élastique du matériau (MPa) \\
\hline$R_{\mathrm{r}}$ & $\begin{array}{l}\text { Résistance à la rupture par traction } \\
\text { du matériau }(\mathrm{MPa})\end{array}$ \\
\hline$r_{\mathrm{p}}$ & Rayon de la bille de brunissage (mm) \\
\hline$t_{i-1}$ & Temps enregistré après l'essai antérieur (min) \\
\hline$V$ & Vitesse de broche en formage $\left(\mathrm{m} \cdot \mathrm{min}^{-1}\right)$ \\
\hline$\Delta m$ & Perte de masse $(\mathrm{g})$ \\
\hline$\Delta t$ & Durée de l'essai (min) \\
\hline
\end{tabular}

couches superficielles et agissent aussi bien sur les propriétés géométriques que physiques.

Les paramètres d'entrée affectant les procédés sont variables d'un auteur à l'autre et se résument généralement aux éléments du régime de travail comme la vitesse, l'avance, l'effort de formage et le nombre de passes [8,10-12]. Bien souvent on introduit d'autres paramètres tels que : la profondeur de pénétration [8], le temps de l'opération [10] ou même l'état initial des couches superficielles [13]. La forme et la géométrie de l'outil sont parfois prises en compte $[8,14]$.

Les réponses de sortie sont beaucoup plus axées sur l'aspect de surface $[1,9,15]$, l'état des contraintes résiduelles en surface $[10,13,15]$ et plus particulièrement la résistance des couches superficielles. D'autres résultats peuvent être attendus des deux procédés tels que la capacité portante, la tolérance dimensionnelle [16] ou la tolérance de forme [17].

Certains travaux ont montré qu'il est possible d'atteindre sous certaines conditions des résultats intéressants à l'égard de propriétés mécaniques telles que la rugosité et la dureté de surface $[8,14,15]$ ou la résistance à l'usure $[3,4]$. Outre ces influences positives, le brunissage et le galetage induisent des contraintes résiduelles de compression dont les valeurs maximales peuvent être localisées à une profondeur de 40 à $200 \mu \mathrm{m}$ [10,18-20]. Il en résulte une bonne résistance à l'initiation et/ou la propagation des fissures [18] et par conséquent une amélioration de la tenue en fatigue 10 à $300 \%$ [19,21,22]. Toutefois, les résultats obtenus sont étroitement liés aux conditions de travail parfois limitées par la conception des outils $[6,17]$, le matériau à traiter $[17,18]$ et l'état initial de ces couches à partir duquel le traitement est plus efficace $[8,20]$.

\section{Matériau et techniques expérimentales}

\subsection{Le matériau}

Le matériau étudié est un acier de nuance $R \mathrm{~b}$. Cet acier de qualité standard a été livré par l'entreprise Arcelor Mittal Steel (Annaba, Algérie) à l'état brut et vieilli naturellement.

Une analyse chimique basée sur l'absorption atomique a révélé la composition chimique suivante :

$0,34 \% \mathrm{C} ; 1,25 \% \mathrm{Mn} ; 0,16 \% \mathrm{Si}$; traces de $\mathrm{S}$ et $\mathrm{P}$.

Un essai de traction appliqué sur un barreau prélevé du matériau sus désigné a permis d'enregistrer les caractéristiques de traction suivantes:

$R_{\mathrm{e}}=447,4 \mathrm{MPa}, R_{\mathrm{r}}=612,5 \mathrm{MPa}$ et $A \%=20,6 \%$.

Les éprouvettes, sous forme de barreaux cylindriques, ont été préparées par tournage avec un outil en carbure métallique de nuance M20. Les couches superficielles résultant du tournage sont caractérisées par une rugosité $R_{\mathrm{a}}=1,9$ à $3,64 \mu \mathrm{m}$ et une dureté $H R B=80$ à 86 .

Après tournage, l'éprouvette est scindée en deux parties. La deuxième partie est destinée selon le cas à subir le brunissage ou le galetage. Enfin sur chacune des deux parties on applique l'essai d'usure.

\subsection{Dispositifs et modes opératoires}

\subsubsection{Dispositif de traitement mécanique}

Les opérations de brunissage et galetage peuvent être effectuées au moyen d'un seul dispositif spécialement conçu à cet effet; seule la partie active est à changer selon le cas. Ce dispositif présente l'avantage d'une simplicité de montage sur la tourelle d'un tour universel (Fig. 1a). Toutefois, le brunissage avec sa pointe en diamant de rayon $r_{\mathrm{p}}=1,5 \mathrm{~mm}$ simule une opération de chariotage; le mouvement de formage est obtenu par la rotation de la pièce, celui de l'avance est communiqué à l'outil par l'intermédiaire du chariot longitudinal (Fig. 1b).

Le galetage avec galet tournant de diamètre $d_{\mathrm{G}}=$ $36 \mathrm{~mm}$ simule une opération de rectification cylindrique; de ce fait il nécessite en plus un mouvement rotatif du galet. Pour les deux procédés, le mouvement de pénétration se traduit par l'action de la pointe en diamant ou du galet tournant qui exerce sur la pièce un effort $P$ de formage préalablement réglé sur le dispositif.

Le brunissage, tout comme le galetage, est effectué dans des conditions de lubrification sous un régime recommandé en fonction de la dureté initiale [23]. Ce régime est défini par : $V=10$ à $80 \mathrm{~m} \cdot \mathrm{min}^{-1} ; f=0,05$ à $0,1 \mathrm{~mm} \cdot \mathrm{tr}^{-1} ; P=5$ à 20 daN et $i=1$ à 3 passes. Afin d'apprécier l'influence de ces paramètres, les essais ont été conduits sous différents régimes dont la combinaison des paramètres (Tab. 1) est dictée par la méthode multifactorielle de planification des expériences [24,25].

\subsubsection{Essai d'usure}

Le but de ces essais est d'estimer la résistance du matériau à l'usure, à l'issue du chariotage ou après 


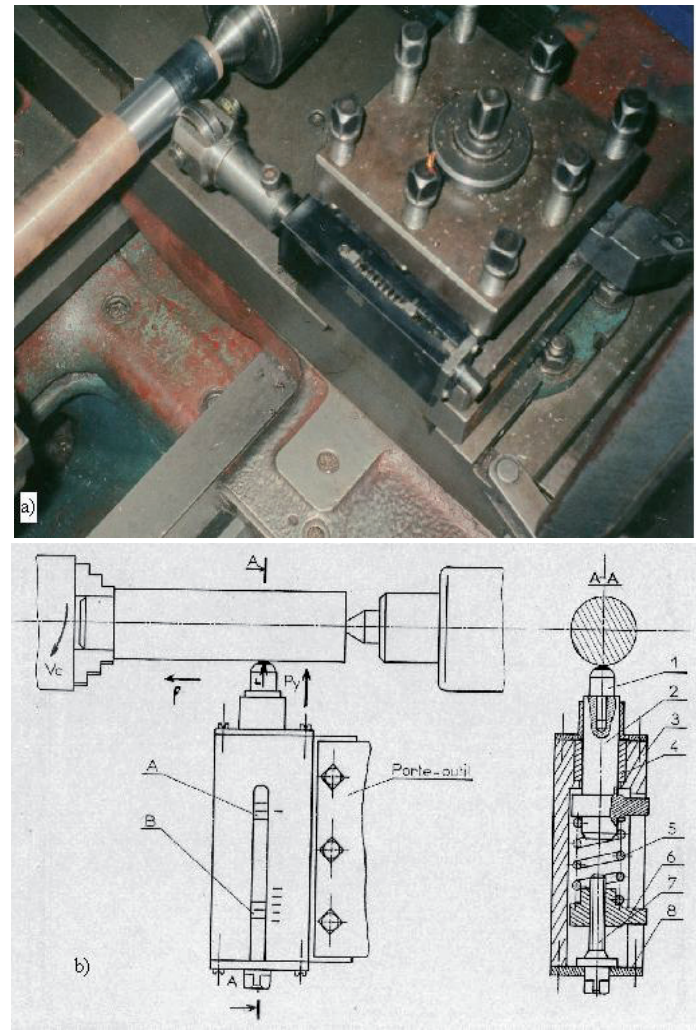

Fig. 1. Dispositif de formage a) photo du montage sur tour, b) schéma de l'opération de brunissage 1) tige; 2) mandrin; 3) corps ; 4) manchon guide ; 5) ressort ; 6) butée ; 7) vis de réglage ; 8) plateau.

Tableau 1. Régimes de travail en traitement mécanique.

\begin{tabular}{|c|c|c|c|c|c|}
\hline \multirow{2}{*}{$\begin{array}{c}\mathrm{N}^{\circ} \\
\text { Essai }\end{array}$} & \multicolumn{5}{|c|}{ Paramètres } \\
\cline { 2 - 6 } & $\begin{array}{c}V \\
\left(\mathrm{~min}^{-1}\right)\end{array}$ & $\begin{array}{c}n \\
\left(\mathrm{tr}_{\text {min }}^{-1}\right)\end{array}$ & $\begin{array}{c}P \\
(\mathrm{kgf})\end{array}$ & $\begin{array}{c}f\left(\mathrm{~mm}_{\mathrm{tr}}^{-1}\right) \\
(\mathrm{kgf})\end{array}$ & $\begin{array}{c}i \\
(\text { passe })\end{array}$ \\
\hline 01 & 10 & 180 & 20 & 0,075 & 2 \\
\hline 02 & 45 & 710 & 20 & 0,075 & 2 \\
\hline 03 & 80 & 1000 & 20 & 0,075 & 2 \\
\hline 04 & 45 & 710 & 20 & 0,05 & 2 \\
\hline 05 & 45 & 710 & 20 & 0,075 & 2 \\
\hline 06 & 45 & 710 & 20 & 0,100 & 2 \\
\hline 07 & 45 & 710 & 05 & 0,075 & 2 \\
\hline 08 & 45 & 710 & 15 & 0,075 & 2 \\
\hline 09 & 45 & 710 & 20 & 0,075 & 2 \\
\hline 10 & 45 & 710 & 15 & 0,075 & 1 \\
\hline 11 & 45 & 710 & 15 & 0,075 & 2 \\
\hline 12 & 45 & 710 & 15 & 0,075 & 3 \\
\hline
\end{tabular}

avoir subi l'un ou l'autre des procédés de formage. Le phénomène d'usure a lieu par abrasion à trois corps dans la mesure où on injecte dans la zone de contact outilpièce des grains d'abrasif véhiculés par l'huile de lubrification. L'essai s'effectue sur un tour parallèle au moyen d'un dispositif (Fig. 2) équipé d'un roulement qui exerce une légère pression sur la pièce. Une fois le contact établi,
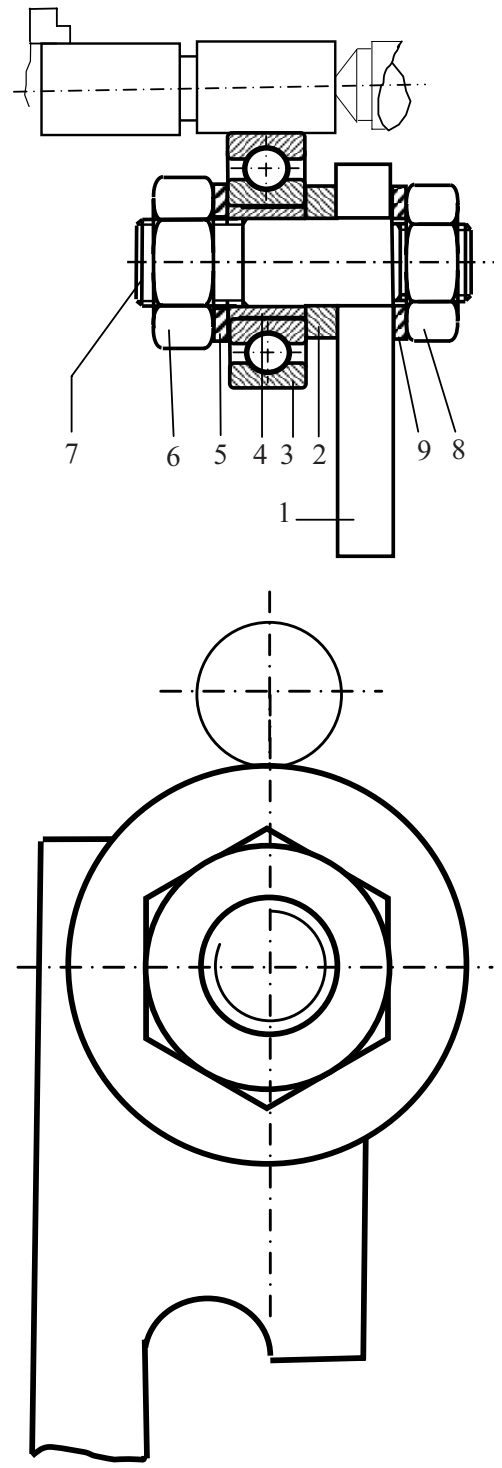

Fig. 2. Dispositif d'essai d'usure. 1) corps ; 2) bague; 3) roulement ; 4) bague ; 5) rondelle de serrage ; 6) écrou M8 ; 7) axe ; 8) écrou M8;9) rondelle.

le roulement est entraîné en rotation résultant d'une friction avec la pièce dont la fréquence de rotation a été fixée à 500 tr.min ${ }^{-1}$. À chaque essai, on fixe le temps et on effectue la pesée de l'éprouvette à l'aide d'une balance de précision.

On enregistre alors la perte de masse $\Delta m$ en surface qui représente la différence entre la masse $m_{i-1}$ avant et $m_{i}$ après l'essai. La valeur cumulée de $\Delta m$ est évaluée par l'expression (1) :

$$
\Delta m=m_{i-1}-m_{i}+\Delta m_{i-1}
$$

La valeur de $\Delta m$ caractérise en effet l'usure résultant du contact outil-abrasif-pièce pour un temps donné. Les éprouvettes se présentant aux essais d'usure ont été sélectionnées selon les meilleures rugosités et duretés résultant de chacun des deux procédés de formage. 
Tableau 2. Effet du brunissage et du galetage sur la rugosité et la dureté.

\begin{tabular}{|c|c|c|c|c|c|c|c|c|c|c|}
\hline \multirow[b]{2}{*}{$\mathrm{N}^{\circ}$} & \multicolumn{5}{|c|}{ Rugosité $R_{\mathrm{a}}[\mu \mathrm{m}]$} & \multicolumn{5}{|c|}{ Dureté [HRB] } \\
\hline & $\begin{array}{l}\text { Après } \\
\text { Tour. }\end{array}$ & $\begin{array}{c}\text { Après } \\
\text { Brunis. }\end{array}$ & $\begin{array}{c}\text { Écart } \\
(\%)\end{array}$ & $\begin{array}{l}\text { Après } \\
\text { Galet. }\end{array}$ & $\begin{array}{c}\text { Écart } \\
(\%)\end{array}$ & $\begin{array}{l}\text { Après } \\
\text { Tour. }\end{array}$ & $\begin{array}{c}\text { Après } \\
\text { Brunis. }\end{array}$ & $\begin{array}{c}\text { Écart } \\
(\%)\end{array}$ & $\begin{array}{l}\text { Après } \\
\text { Galet. }\end{array}$ & $\begin{array}{c}\text { Écart } \\
(\%)\end{array}$ \\
\hline 01 & \multirow{3}{*}{2,4} & 1,9 & 20,83 & 0,32 & 86,66 & \multirow{3}{*}{82} & 87 & 6,09 & 86 & 4,87 \\
\hline 02 & & 1,6 & 33,33 & 0,29 & 87,91 & & 85 & 3,65 & 85 & 3,65 \\
\hline 03 & & 1,5 & 37,50 & 0,22 & 90,83 & & 84 & 2,43 & 84 & 2,43 \\
\hline 04 & \multirow{3}{*}{1,8} & 1,1 & 38,88 & 0,22 & 87,77 & \multirow{3}{*}{83} & 89 & 7,22 & 85 & 2,40 \\
\hline 05 & & 1,4 & 22,22 & 0,25 & 86,11 & & 85 & 2,40 & 85 & 2,40 \\
\hline 06 & & 1,6 & 11,11 & 0,37 & 79,44 & & 84 & 1,20 & 85 & 2,40 \\
\hline 07 & \multirow{3}{*}{3,0} & 1,1 & 63,33 & 1,84 & 38,66 & \multirow{3}{*}{83} & 85 & 2,40 & 83 & 0,00 \\
\hline 08 & & 0,9 & 70,00 & 0,39 & 87,00 & & 86 & 3,61 & 85 & 2,40 \\
\hline 09 & & 1,1 & 63,33 & 0,25 & 91,66 & & 90 & 8,43 & 87 & 4,82 \\
\hline 10 & \multirow{3}{*}{1,9} & 1,8 & 05,26 & 0,46 & 75,78 & \multirow{3}{*}{79} & 84 & 6,32 & 82 & 3,79 \\
\hline 11 & & 1,4 & 26,31 & 0,30 & 84,21 & & 85 & 7,60 & 83 & 5,06 \\
\hline 12 & & 1,3 & 11,40 & 0,24 & 87,36 & & 87 & 10,12 & 84 & 6,32 \\
\hline
\end{tabular}

\section{Résultats}

\subsection{Effets des traitements}

L'aspect initial des couches superficielles est le résultat d'une opération de tournage dont le régime de coupe optimal a été dégagé par fitting pour cibler une rugosité initiale de $3,2 \mu \mathrm{m}$. Cet état géométrique est recommandé $[5,8,23]$ pour une meilleure efficacité du traitement. D'ailleurs, les diverses combinaisons des paramètres des traitements ont permis de révéler une rugosité $R_{\mathrm{a}}$ comprise entre 0,22 et $0,9 \mu \mathrm{m}$ et une dureté $H R B$ entre 87 et 90 (Tab. 2). Toutefois, le maximum d'effet apporté à chacune des deux caractéristiques (Fig. 3) résulte d'un régime de travail spécifique à chaque traitement.

Lors du brunissage, fixant $V=45 \mathrm{~m} \cdot \mathrm{min}^{-1}, P=$ 15 daN et $f=0,075 \mathrm{~mm} \cdot \mathrm{tr}^{-1}$, le traitement en 3 passes, permet d'améliorer la dureté d'environ 10-12 \%. En revanche, si le traitement est limité à 2 passes, la rugosité $R_{\text {a }}$ est améliorée d'environ $70 \%$.

Lors du galetage, le régime le plus avantageux visà-vis de $R_{\mathrm{a}}$ et $H R B$ est également défini par les valeurs moyennes de $V$ et $f$. Toutefois, en combinant à ces valeurs un effort de 20 daN en deux passes, la rugosité se voit améliorée de $92 \%$. En contre partie, lorsque l'effort de formage est réduit à 15 daN et lorsque le nombre de passes augmente à 3 , la dureté gagne jusqu'à $6 \%$.

\subsection{Influence du régime de traitement}

La figure 4 illustre l'influence des paramètres du traitement sur le taux d'accroissement de la rugosité après action des deux procédés.

Les deux procédés sont peu sensibles à la variation de la fréquence de rotation ou à la vitesse d'avance (Figs. 4a, b) quoiqu'une augmentation de l'avance diminue l'efficacité du brunissage.
Une augmentation de l'effort de formage et du nombre de passes lors du galetage (Figs. 4c, d) améliore davantage l'aspect géométrique de la surface. Cette amélioration est moins poussée lors du brunissage. Il ne serait donc pas désirable de trop augmenter ces deux paramètres de traitement afin de ne pas minimiser les effets du brunissage.

La figure 5 montre que les courbes illustrant l'influence des paramètres du régime de traitement sur la dureté présentent les mêmes allures pour les deux traitements.

Il ressort de l'analyse de ces courbes que le taux d'accroissement de la dureté diminue avec l'augmentation de la fréquence de rotation (Fig. 5a) ou même la vitesse d'avance (Fig. 5b). Par contre, l'augmentation de l'effort de formage (Fig. 5c) et du nombre de passes (Fig. 5d) contribue à améliorer la dureté, cette amélioration étant plus marquée à l'issue du brunissage.

\subsection{Résistance à l'usure}

Les essais ont été réalisés pendant une durée spécifique de $60 \mathrm{~min}$. La perte relative de masse $\left(\Delta \mathrm{m} / \mathrm{m}_{0}\right)$ enregistrée sur les échantillons à l'état tourné est comprise entre 1,94 et 3,32 \%. Après galetage, la perte de masse représente $0,21 \%$ (Tab. 3) alors qu'à l'issue du brunissage ce paramètre est réduit à $0,05 \%$ (Tab. 4). Dans ce cas, les deux procédés améliorent la tenue à l'usure, l'amélioration étant mieux ressentie pour le brunissage (98\%) que chez le galetage (89\%) (Fig. 6).

L'évolution de l'usure en fonction du temps est illustrée par la figure 7. L'allure des courbes révèle les lois classiques d'usures [1] où on peut mettre en évidence les trois stades classiques d'usure.

Le premier stade caractéristique du rodage des aspérités de rugosité se manifeste jusqu'aux 20 premières minutes de l'essai, ce stade étant plus accéléré lors du galetage. Le second stade représente l'usure systématique normale dont l'évolution au cours du temps est plus ou moins 


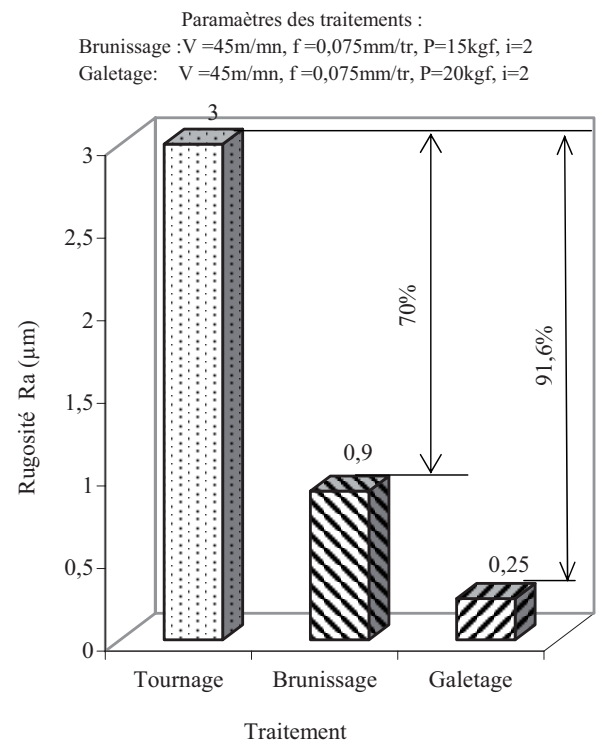

(a)

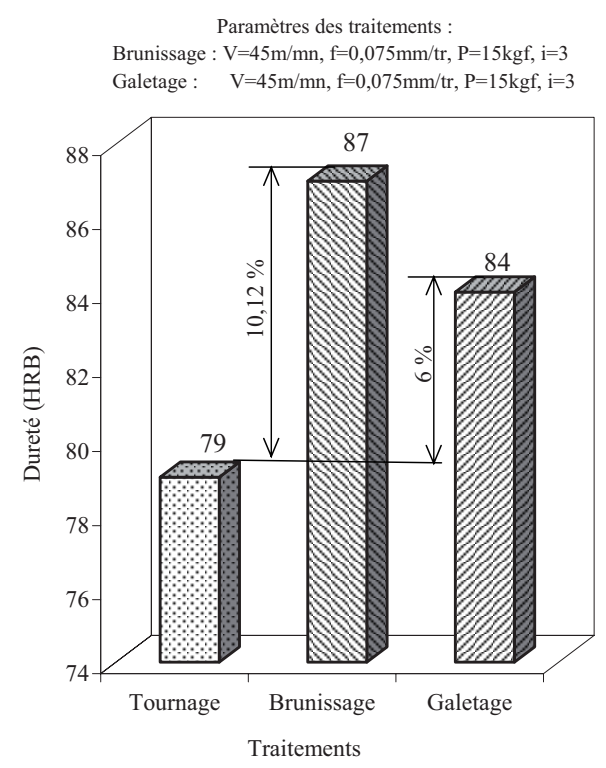

(b)

Fig. 3. Effet des traitements de formage sur : a) la rugosité et b) la dureté.

régie par une loi linéaire. Le troisième stade se distingue au-delà de 60 min d'essai, la perte de masse s'accélère de façon importante, on estime donc que l'usure serait entrée dans son domaine catastrophique. Le matériau se dégrade de façon très sévère au contact de l'outil.

\section{Discussion}

\subsection{Analyse des résultats}

Les deux procédés de formage mettent en œuvre la déformation plastique des couches superficielles et modifient certains aspects physique et géométrique de celles-ci. Il en résulte de cette plastification que les crêtes des aspérités d'usinage sont aplaties pour mieux remplir les creux des sillons. Ces aspérités nivelées par le passage des outils laissent derrière elles une surface totalement polie avec une rugosité comparable à celle fournie par les usinages de finition classiques [4,7].

L'importance d'une part du rayon du galet devant celui de la pointe de brunissage, d'autre part de l'aire de contact outil-pièce font du galet un atout pour mieux aplatir les aspérités et améliorer davantage l'état de surface (diminution de $R_{\mathrm{a}}$ voisine de $90 \%$ ). Cette amélioration est peu ressentie lors du brunissage dans le sens où la diminution de la rugosité était de $70 \%$. En effet, la pointe en diamant de l'outil attaque la pièce de la même manière qu'un outil coupant. L'application ponctuelle de l'outil est peu efficace par rapport au galetage. Par ailleurs, il ressort de l'analyse des résultats que l'obtention d'une rugosité optimale dépend d'une part de l'état initial de surface (résultat du tournage) et d'autre part d'un régime spécifique appliqué lors du traitement.

En effet le meilleur résultat fournissant la plus petite rugosité avant et après traitement a été enregistré à partir d'une rugosité initiale de l'ordre de $3 \mu \mathrm{m}$ (Fig. 3a), cette valeur étant située dans le même ordre de grandeur que celle préconisée par certains travaux [5, 8, 12]. Elle concerne aussi bien le galetage que le brunissage.

En vue d'obtenir une rugosité optimale, les régimes spécifiques aux deux traitements se distinguent entre eux seulement par le choix de l'effort de formage $P$. Le meilleur effet est acquis pour une force de 15 daN lors du galetage (Fig. 4c). La faible étendue de contact outilpièce lors du brunissage semble favoriser la pénétration de l'outil dans la pièce au fur et à mesure qu'on augmente l'effort $P$. Par conséquent, la pièce se consolide davantage par suite d'un taux élevé d'écrouissage induit par la déformation plastique des couches superficielles. Le durcissement de la surface provoque sa détérioration par écaillage au contact de l'outil et altère son aspect qui préserve quand même l'avantage d'être meilleur par rapport à l'état initial.

Lors du galetage, la largeur du galet favorise une grande aire de contact ce qui aura pour effet d'une part, un allègement de la pression de contact et d'autre part, un écoulement régulier des crêtes dans les creux des aspérités. De ces effets, résulte un meilleur état de surface caractérisé par une faible rugosité. Ceci dit, tant que l'effort de formage est faible $(P<10$ daN $)$, l'action du brunissage demeure la plus avantageuse à l'égard de la rugosité. C'est pourquoi la figure $4 \mathrm{c}$ présente une interaction entre les deux procédés.

Une augmentation de la fréquence de rotation dans la gamme choisie des vitesses contribue à augmenter le taux d'amélioration de la rugosité (Fig. 4a). C'est que la température dans la zone de formage augmente avec la vitesse avant de se stabiliser à un certain moment. Les couches superficielles tendent à prendre un aspect de plus en plus visqueux ce qui réduit le frottement entre l'outil et la pièce et par conséquent, la rugosité s'améliore davantage. Le meilleur résultat est obtenu pour le brunissage à cause de la meilleure 


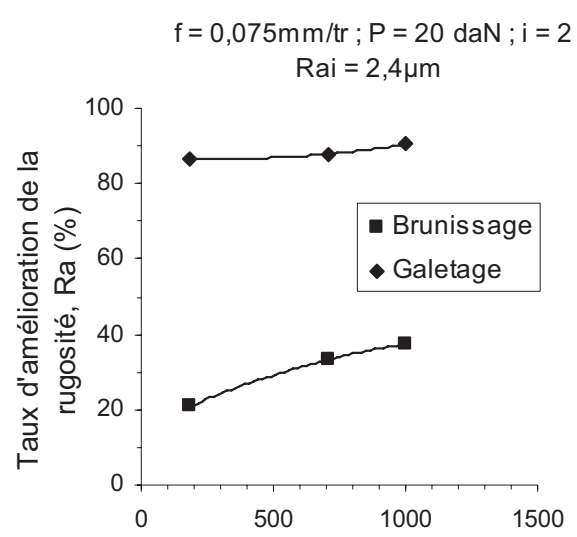

a)

$$
\begin{gathered}
\mathrm{n}=710 \mathrm{tr} / \mathrm{min} ; \mathrm{f}=0,075 \mathrm{~mm} / \mathrm{tr} ; \mathrm{i}=2 \\
\text { Rai }=3 \mu \mathrm{m}
\end{gathered}
$$

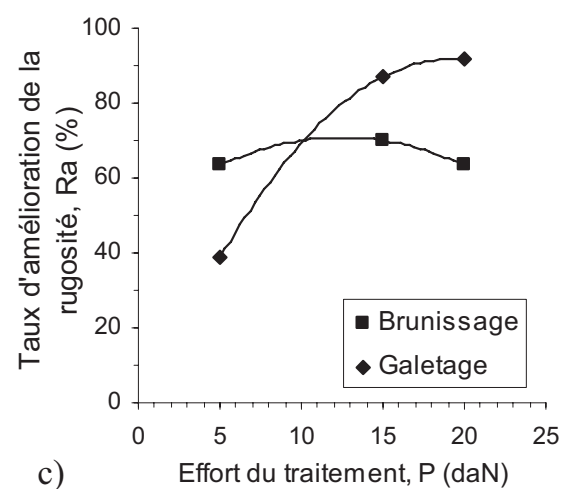

$\mathrm{n}=710 \mathrm{tr} / \mathrm{min} ; \mathrm{P}=20 \mathrm{daN} ; \mathrm{i}=2$

Rai $=1,8 \mu \mathrm{m}$

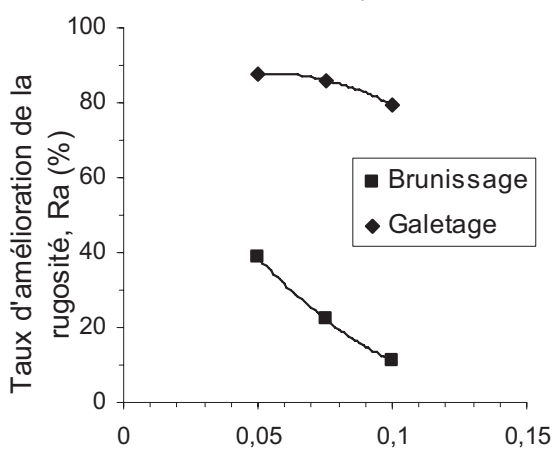

b) Avance du traitement, $\mathrm{f}(\mathrm{mm} / \mathrm{tr})$ $\mathrm{n}=710 \mathrm{tr} / \mathrm{min} ; \mathrm{f}=0,075 \mathrm{~mm} / \mathrm{tr} ; \mathrm{P}=15 \mathrm{daN}$ Rai $=1,9 \mu \mathrm{m}$

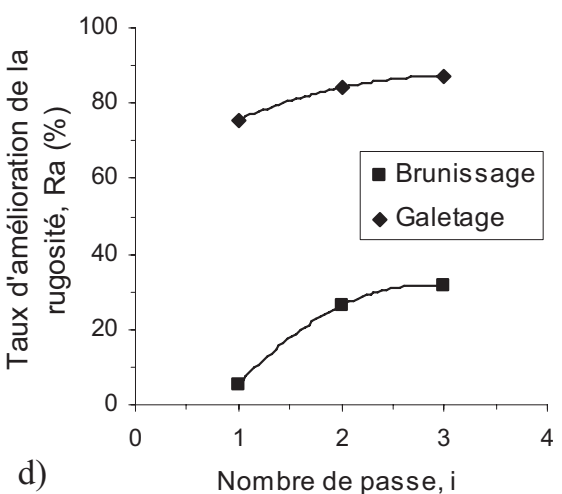

Fig. 4. Influence des éléments du régime de traitement sur la rugosité.

rigidité en service de l'outil. Toutefois, l'augmentation de la fréquence de rotation n'est pas souhaitable au-delà de certaines valeurs à cause d'une part, de l'instabilité de l'outil, la faible déformation plastique et l'insuffisance de temps de lubrification [15], d'autre part, du phénomène de broutement qui est à l'origine de l'altération de la surface [14].

Une augmentation de l'avance (Fig. 4b) augmente le pas des sillons de l'outil ce qui tend à altérer la rugosité [1]. Le taux d'amélioration est plus affecté par la baisse lors du brunissage. Le galetage offre dans ce cas le meilleur résultat grâce à la forme de l'outil (donc du contact outil-pièce) qui travaille par génération linéaire et au mouvement de rotation supplémentaire de sa partie active lesquels contribuent à un meilleur nivellement des aspérités.

L'augmentation du nombre de passes contribue à augmenter le taux d'amélioration de la rugosité (Fig. 4d). Il semble que l'effet du nombre de passes est le même que celui de l'effort de formage. Toutefois, il n'est pas désirable de trop augmenter le nombre de passes de crainte de sursaturer le matériau en écrouissage au risque de détériorer la surface une fois qu'on aurait dépassé l'aptitude à la déformation plastique du matériau [9].

\section{2 Évolution de la dureté}

L'amélioration de la dureté est le résultat d'un écrouissage des couches superficielles par suite de leur plastification. Le brunissage par sa pointe en diamant écrouit mieux le matériau par rapport au galetage bien que les duretés optimales résultant des deux procédés soient dans le même ordre de grandeur (Fig. 3b). Les valeurs optimales avec le même régime de traitement.

Lors du brunissage le régime spécifique à la dureté optimale diffère de celui relatif à la rugosité seulement par le nombre de passes qui change de 2 à 3 .

Par contre, pour le galetage et selon que l'on mise sur la rugosité ou sur la dureté optimale, on doit prendre en considération les deux paramètres décisifs $P$ et $i$. L'influence de ces deux paramètres sur la dureté est régie par les mêmes phénomènes. L'augmentation de l'effort de formage tout comme l'augmentation du nombre de passes plastifie davantage le matériau d'où sa consolidation par écrouissage. Par sa pointe en diamant, l'outil de brunissage affecte beaucoup plus en profondeur les couches superficielles et fournit donc le meilleur résultat. Par ailleurs, comme il est recommandé [9], on doit limiter ces valeurs pour ne pas sursaturer en écrouissage le matériau de crainte de développer des micro-fissurations du matériau. 
$\mathrm{f}=0,075 \mathrm{~mm} / \mathrm{rev} ; \mathrm{P}=20 \mathrm{daN} ; \mathrm{i}=2$ $\mathrm{HRBi}=82$

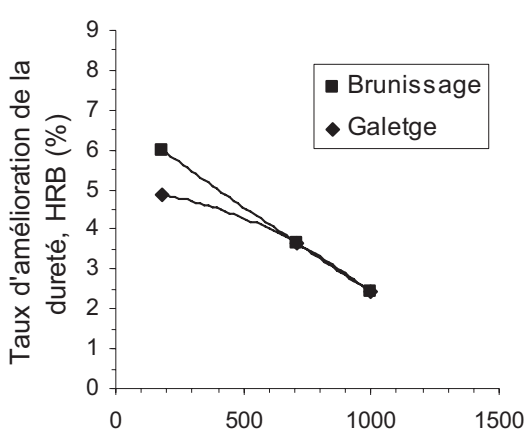

a) Vitesse du traitement, $\mathrm{n}(\mathrm{tr} / \mathrm{min})$

$\mathrm{n}=710 \mathrm{tr} / \mathrm{min} ; \mathrm{f}=0,075 \mathrm{~mm} / \mathrm{tr} ; \mathrm{i}=2$ $\mathrm{HRBi}=83$

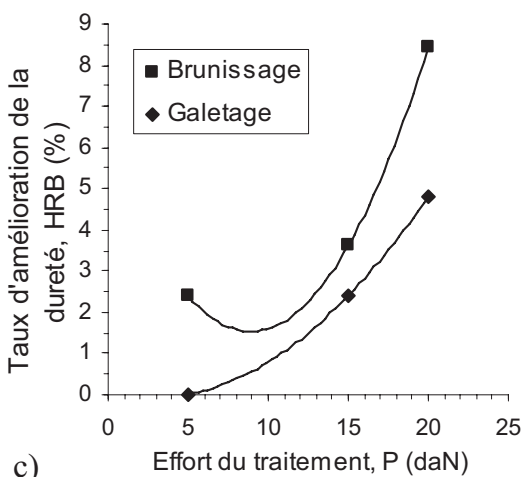

$\mathrm{n}=710 \mathrm{tr} / \mathrm{min} ; \mathrm{P}=20 \mathrm{daN} ; \mathrm{i}=2$ $\mathrm{HRBi}=83$

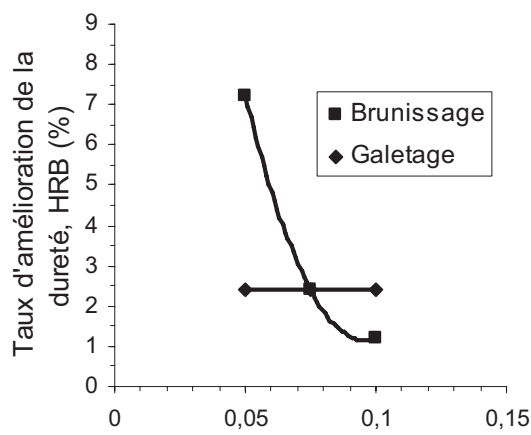

b) Avance du traitement, $\mathrm{f}(\mathrm{mm} / \mathrm{tr})$ $\mathrm{n}=710 \mathrm{tr} / \mathrm{min} ; \mathrm{f}=0,075 \mathrm{~mm} / \mathrm{tr} ; \mathrm{P}=15 \mathrm{daN}$ $\mathrm{HRBi}=79$

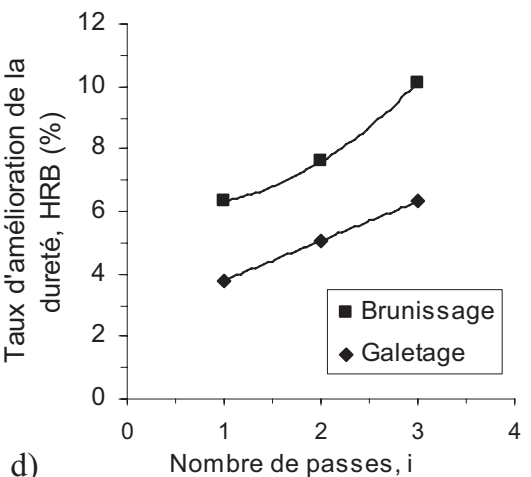

d)

Fig. 5. Influence des éléments du régime de traitement sur la dureté.

Tableau 3. Résultats des essais d'usure après galetage.

\begin{tabular}{|c|c|c|c|c|c|c|c|c|}
\hline \multirow[t]{2}{*}{$\mathrm{N}^{\circ}$} & \multirow[t]{2}{*}{$\begin{array}{c}\Delta t_{i} \\
{[\mathrm{~min}]}\end{array}$} & \multirow[t]{2}{*}{$\begin{array}{c}t_{i-1}+\Delta t \\
{[\mathrm{~min}]}\end{array}$} & $\begin{array}{l}m_{i} \\
(\mathrm{~g})\end{array}$ & $\begin{array}{c}\Delta m_{i} \\
(\mathrm{~g})\end{array}$ & $\begin{array}{c}\Delta m_{i} / m_{0} \\
(\%)\end{array}$ & $\begin{array}{l}m_{i} \\
(\mathrm{~g})\end{array}$ & $\begin{array}{c}\Delta m_{i} \\
(\mathrm{~g})\end{array}$ & $\begin{array}{c}\Delta m_{i} / m_{0} \\
(\%)\end{array}$ \\
\hline & & & \multicolumn{3}{|c|}{ Tournage $: m_{0}=77,8380 \mathrm{~g}$} & \multicolumn{3}{|c|}{ Galetage $: m_{0}=78,0053 \mathrm{~g}$} \\
\hline 01 & 10 & 10 & 77,4960 & 0,3420 & 0,440 & 78,0024 & 0,0029 & 0,004 \\
\hline 02 & 5 & 15 & 77,3496 & 0,4884 & 0,627 & 78,0010 & 0,0043 & 0,005 \\
\hline 03 & 5 & 20 & 77,0960 & 0,7420 & 0,953 & 77,9472 & 0,0581 & 0,074 \\
\hline 04 & 5 & 25 & 77,0842 & 0,7538 & 0,970 & 77,9335 & 0,0718 & 0,092 \\
\hline 05 & 5 & 30 & 76,9790 & 0,8590 & 1,103 & 77,9243 & 0,0810 & 0,104 \\
\hline 06 & 10 & 40 & 76,8520 & 0,9860 & 1,266 & 77,9001 & 0,1052 & 0,134 \\
\hline 07 & 5 & 45 & 76,4350 & 1,4030 & 1,802 & 77,8947 & 0,1106 & 0,142 \\
\hline 08 & 5 & 50 & 76,4240 & 1,4140 & 1,816 & 77,8625 & 0,1428 & 0,183 \\
\hline 09 & 5 & 55 & 76,3974 & 1,4406 & 1,850 & 77,8909 & 0,1444 & 0,185 \\
\hline 10 & 5 & 60 & 76,3231 & 1,5149 & 1,946 & 77,8380 & 0,1673 & 0,214 \\
\hline
\end{tabular}

L'augmentation de la vitesse de rotation et de l'avance de travail réduit les effets bénéfiques des deux traitements à l'égard de la dureté (Figs. 5a, b). Il semble qu'à vitesse élevée, le taux de déformation du matériau est insuffisant pour le consolider suffisamment. Il en est de même pour l'avance qui est liée à la vitesse de rotation par la chaîne cinématique de la machine-outil.

Avec l'augmentation de la vitesse, le brunissage se comporte de façon analogue au galetage, alors qu'en augmentant l'avance, on remarque une interaction entre les procédés. Pour les faibles avances, le galetage est plus sensible à la dureté alors qu'il l'est moins pour des valeurs importantes.

\subsection{Tenue à l'usure}

L'amélioration de la tenue à l'usure (Fig. 6) par les deux traitements semble être une conséquence directe du 
Tableau 4. Résultats des essais d'usure après brunissage.

\begin{tabular}{|c|c|c|c|c|c|c|c|c|}
\hline $\mathrm{N}^{\circ}$ & $\begin{array}{c}\Delta t_{i} \\
(\mathrm{~min})\end{array}$ & $\begin{array}{c}t_{i-1}+\Delta t \\
(\mathrm{~min})\end{array}$ & $\begin{array}{c}m_{i} \\
(\mathrm{~g})\end{array}$ & $\begin{array}{c}\Delta m_{i} \\
(\mathrm{~g})\end{array}$ & $\begin{array}{c}\Delta m_{i} / m_{0} \\
(\%)\end{array}$ & $\begin{array}{c}m_{i} \\
(\mathrm{~g})\end{array}$ & $\begin{array}{c}\Delta m_{i} \\
(\mathrm{~g})\end{array}$ & $\begin{array}{c}\Delta m_{i} / m_{0} \\
(\%)\end{array}$ \\
\cline { 4 - 9 } & & & \multicolumn{2}{|c|}{ Tournage $: m_{0}=71,8800 \mathrm{~g}$} & \multicolumn{2}{|c|}{ Brunissage $: m_{0}=71,9180 \mathrm{~g}$} \\
\hline 01 & 10 & 10 & 70,9850 & 0,8950 & 1,245 & 71,9153 & 0,0027 & 0,004 \\
\hline 02 & 5 & 15 & 70,6820 & 1,1980 & 1,666 & 71,9129 & 0,0051 & 0,007 \\
\hline 03 & 5 & 20 & 70,1933 & 1,6867 & 2,346 & 71,9123 & 0,0057 & 0,008 \\
\hline 04 & 5 & 25 & 70,0831 & 1,7969 & 2,500 & 71,9104 & 0,0076 & 0,010 \\
\hline 05 & 5 & 30 & 70,0725 & 1,8075 & 2,514 & 71,9100 & 0,0080 & 0,011 \\
\hline 06 & 10 & 40 & 69,9730 & 1,9070 & 2,653 & 71,9097 & 0,0083 & 0,011 \\
\hline 07 & 5 & 45 & 69,7402 & 2,1398 & 2,977 & 71,9031 & 0,0149 & 0,021 \\
\hline 08 & 5 & 50 & 69,6402 & 2,2398 & 3,116 & 71,8980 & 0,0200 & 0,028 \\
\hline 09 & 5 & 55 & 69,5382 & 2,3418 & 3,258 & 71,8913 & 0,0267 & 0,037 \\
\hline 10 & 5 & 60 & 69,4892 & 2,3908 & 3,326 & 71,8800 & 0,0380 & $\mathbf{0 , 0 5 3}$ \\
\hline
\end{tabular}

Régime des traitements :

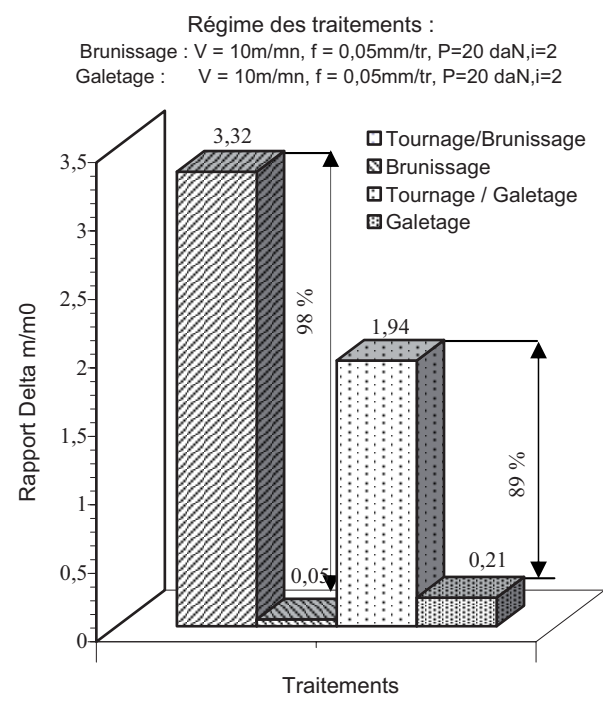

Fig. 6. Effet du traitement sur la tenue à l'usure.

meilleur poli de surface et la dureté des couches superficielles déduits des deux procédés $[3,4,22]$. La courbe de perte de masse (Fig. 7) résultant du brunissage est située en dessous de celle fournie par le galetage, ceci explique que le procédé de brunissage offre le meilleur résultat à l'égard de la résistance à l'usure qui a enregistré une amélioration jusqu'à $98 \%$ dans ce cas là.

Les couches superficielles traitées par brunissage étant les plus dures que celles galetées font qu'elles résistent mieux à l'usure. Le meilleur poli de surface résultant du galetage est interprété par le stade de rodage sur la courbe d'usure qui se confond avec celui au brunissage.

Dans le stade d'usure normale, les deux courbes évoluent de la même façon. La courbe relative au brunissage évolue avec une pente moins raide ce qui confirme la meilleure efficacité de ce procédé. Au-delà de ce stade, la couche écrouie se dégrade au contact de l'outil et l'usure s'intensifie de façon catastrophique.

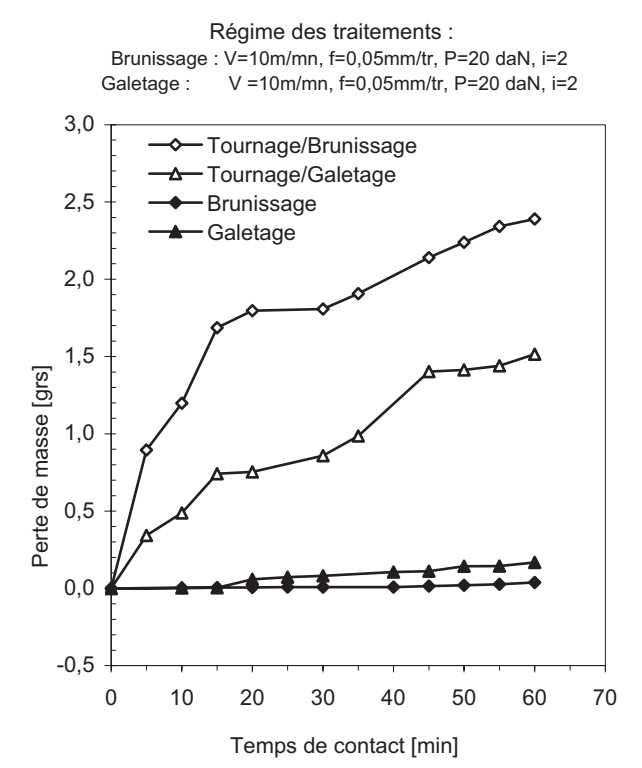

Fig. 7. Évolution de la perte de masse au cours du temps.

\section{Conclusion}

Le galetage et le brunissage sont deux traitements mécaniques de surface à froid par déformation plastique des couches superficielles. Ils offrent un glaçage par refoulement du matériau. Ce refoulement engendre un fluage à la surface du matériau où une onde métallique se forme devant l'outil et régénère une surface nouvelle derrière lui. Les deux procédés améliorent l'état de surface et augmentent la dureté.

La plus faible rugosité est fournie par le galetage; ce résultat est d'autant meilleur que la rugosité initiale est proche de $3 \mu \mathrm{m}$. Alors que le meilleur résultat de la dureté est obtenu par le brunissage. Par ailleurs l'efficacité optimale de chaque procédé est régie par un régime spécifique dont les paramètres importants sont l'effort de formage et le nombre de passes. 
Pour une meilleure rugosité, il est recommandé de limiter le nombre de passes à 2 ; par contre, pour cibler une meilleure dureté, on peut pousser ce paramètre à 3 passes auquel il faut associer à un effort de 15 daN. L'effet de l'effort de formage sur la rugosité entraîne une interaction entre les deux procédés dans les limites de la gamme choisie.

Dans ces conditions d'essai, bien que recherchées pour la productivité et l'économie de l'opération, l'augmentation de la vitesse et de l'avance n'a pas d'influence significative sur la rugosité et la dureté.

Les couches superficielles traitées par galetage et par brunissage se comportent comme des surfaces rodées et présentent une bonne tenue à l'usure. Le brunissage est le plus favorable à celle-ci.

\section{Références}

[1] R. Weil, Techniques d'usinage, Éditions Dunod, Paris, 1971

[2] J. Baillon, Des matériaux, $3^{\mathrm{e}}$ édition, Presses Internationales Polytechniques, Montréal, 2000

[3] A.M. Hassan, A.D. Suleiman, Improvement in the wear resistance of brass components by ball burnishing process, J. Mater. Process. Technol. 96 (1999) 73-80

[4] A.C.E. Mendar, La technique du galetage, Métaux et Déformation 47 (1978) 45-57

[5] J. Lemercier, Emploi rationnel du galetage, Bull. CT de l'Industrie du Décolletage 41 (1976) 5-18

[6] http/www.cogsdill.com, Outils et machines à galeter, Cogsdill-Nuneaton tool products, INC, Tenlons road, Nuneaton, England, 2004

[7] H. Hamadache, K. Chaoui, Mechanical properties improvements of Rb40 steel through ball burnishing process, Séminaire international de génie mécanique, Oran, avril 2002, pp. 425-430

[8] N.H. Loh, S.C. Tam, S. Miyazama, Surface hardening by ball burnishing, Proc. Engng. 23 (1990) 413-417

[9] A. Mahmood Hassan, The effect of ball and roller burnishing on the surface roughness of some non-ferrous metals, J. Mat. Proc. Tech. 72 (1997) 385-391
[10] M. Fattouh, M.M. El-Khabeery, Residual stress distribution in burnishing solution treated and aged $7075 \mathrm{Al}$ alloy, Int. J. Mach. Tools Manufact. 29 (1989) 153-160

[11] V.M. Braslavski, A.A. Baraz, Deformation strengthening of machinery parts, Vestnik Machinostroeniya 63 (1983) 46-51

[12] M.H. El-Axir, An investigation into roller burnishing, Int. J. Mach. Tools Manufact. 40 (2003) 1603-1617

[13] P.I. Kudryavstsev, Surface work-hardening delays fatigue development, Russian Eng. J. LII (1983) 61-65

[14] A.M. Hassan, An investigation into the characteristics of burnished cast Al-Cu alloy, Int. J. Mach. Tools Manufact. 37 (1997) 813-821

[15] M.M. Khabeery, M.H. El-Axir, Experimental techniques for studying the effects of milling roller burnishing parameter on surface integrity, Mach. Tools Manufact. 41 (2001) 1705-1719

[16] K.H. Wang, L.A. Blunt, K.J. Stout, The 3D characterization of the surface topography of the ballizing process, Int. J. Mach. Tools Manufact. 38 (1998) 419-423

[17] M.H. El-Axir, M.M. El Khabeery, Influence of orthogonal burnishing parameters on surface characteristics for various material, J. Mat. Proc. Tech. 132 (2003) 82-89

[18] L. Wagner, Mechanical surface treatments on $\mathrm{Ti}, \mathrm{Al}$ and Mg alloys, Mat. Sce. Ingng. A263 (1999) 210-216

[19] P. Zhang, J. Lindemann, Effect of roller burnishing on high cycle fatigue performance of the high-strength wrought magnesium alloy AZ80, Scr. Mat. 52 (2005) 1011-1015

[20] F. Klocke, J. Lierman, Roller burnishing of hard turned surfaces, Int. J. Mach. Tools Manufact. 38 (1998) 419-423

[21] H.P. Lieurade, La pratique des essais de fatigue, Pyc éditions, Paris, 1982

[22] S. Braham, Modélisation du galetage des vilebrequins, Évolution des contraintes résiduelles sous chargement cycliques, Thèse de doctorat, Palaiseau, Oct. 1991

[23] D.D. Papchev, Procédé de brunissage et son influence sur les propriétés mécaniques, Éditions MIR, Moscou, 1978

[24] E.S. Bojonov, I. Voutchkov, Méthodes statistiques pour la modélisation, Technica, Sofia, 1978

[25] J. Goupy, Plans d'expériences pour surfaces de réponse, Éditions Dunod, 1999 